\title{
Phaco capsulotomy: a technique to prevent the Argentinean Flag Sign
}

\author{
Christopher C Teng \\ Department of Ophthalmology and \\ Visual Science, Yale University School \\ of Medicine, New Haven, CT, USA
}

This article was published in the following Dove Press journal:

Clinical Ophthalmology

6 November 2017

Number of times this article has been viewed

\begin{abstract}
The Argentinean Flag Sign is a complication that occurs during capsulorhexis construction, in which the capsulorhexis extends to the periphery due to lens intumescence. Phaco capsulotomy is a technique in which the phacoemulsification tip is used to simultaneously create the initial tear in the anterior capsule and remove a portion of the intumescent lens, thereby debulking and relieving pressure from the lens and capsule, and preventing the Argentinean Flag Sign. A detailed description of the phaco capsulotomy technique is provided, including applications and potential complications.
\end{abstract}

Keywords: phaco capsulotomy, Argentinean Flag Sign, intumescent cataracts

\section{Introduction}

White cataracts and intumescent cataracts are challenging cases for almost all surgeons. In these eyes, during capsulorhexis creation, the pressure created by the hyper-hydration of lens fibers can cause spontaneous tears in the capsulorhexis that extend to the periphery. This has been termed the Argentinean Flag Sign. Once this occurs, the cataract surgery may become extremely difficult due to poor construction of the capsulorhexis, and possible rupture of posterior capsule.

The Argentinean Flag Sign is the appearance of the stained blue anterior capsule beside the white cataract, which mimics the blue-white-blue pattern of the Argentina flag, and was named by Dr Daniel Mario Perrone. ${ }^{1}$ His video on this complication won awards at the 2000 American Society of Cataract and Refractive Surgery and the European Society of Cataract and Refractive Surgeons video competitions. The blue color of the capsule is the result of staining with Trypan blue, which helps to visualize the anterior capsule in white cataracts.

One method for preventing the Argentinean Flag Sign is by introducing a 27 or 30 -gauge needle on a syringe into an intact anterior capsule. The needle is used to aspirate the liquefied cortex, thereby depressurizing the nucleus, which facilitates a controlled capsulorhexis. ${ }^{2}$ A highly cohesive ophthalmic viscosurgical device (OVD) can be used to pressurize the anterior chamber against the pressure of the intumescent lens, which can facilitate continuous curvilinear capsulorhexis (CCC) completion. ${ }^{3}$ Alternatively, a CCC can be created using a 2-stage technique, which can help prevent unexpected radial tears. ${ }^{4}$ Femtosecond laser-assisted system for capsulotomy has also been reported to have good efficacy for capsulorhexis construction in white cataracts. ${ }^{5}$

Phaco capsulotomy is a technique in which the phacoemulsification probe is introduced through the center of an intact anterior capsule and a portion of the lens is aspirated, thereby depressurizing the capsular bag. This technique has been described in Eyeworld, ${ }^{6}$ and by Mahalingam and Sambhav. ${ }^{7}$ The technique simultaneously creates 
the initial anterior capsule puncture and removes some of the liquefied cortex and nucleus. The automated phaco handpiece debulks and depressurizes the entire lens/capsule apparatus, and removes the impetus for the capsule to tear outwards. This technique can be safely and effectively employed to prevent the Argentinean Flag Sign.

\section{Surgical technique}

The initial steps are identical to a cataract extraction in which blue capsular stain is used. This includes creating a paracentesis, using a capsular stain, injecting highly cohesive and/or dispersive OVD, and creating a main wound. After the main wound is created, the phacoemulsification handpiece is introduced into the eye. The handpiece need not be irrigating upon entry, as there is OVD present in the anterior chamber. The bevel of the tip may be facing up or down, depending on surgeon preference (Figure 1). Next, the phaco handpiece should be directed in a downward position and situated over the center of the anterior capsule.

The next sequence of events occurs simultaneously. The anterior capsule is punctured with the phaco tip, which creates the initial anterior capsule puncture, and the phaco tip is introduced into the lens (Figure 1). The foot pedal of the phacoemulsification unit is depressed to position 2 , which introduces irrigation and aspiration at a fixed rate. The milky cortex will become visible, and will be quickly aspirated into the handpiece. The phaco tip is embedded into the nucleus and the foot pedal is depressed to sculpt

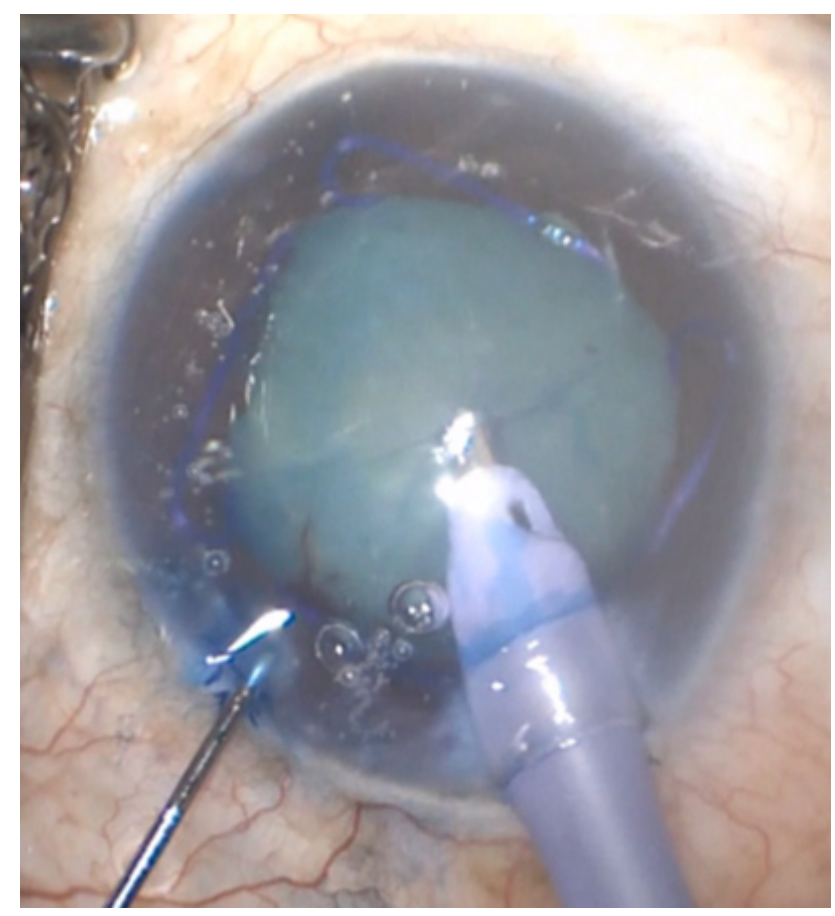

Figure I Phaco tip puncturing an intact anterior capsule.

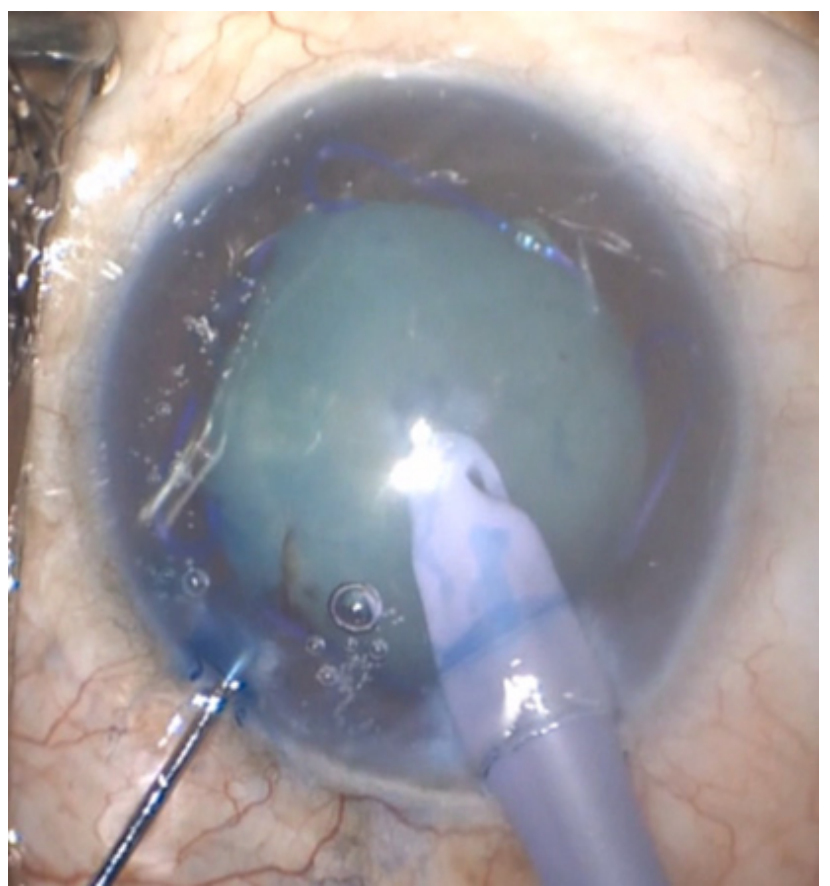

Figure 2 Phaco tip aspirating and debulking the nucleus.

mode, and a portion of the lens nucleus is phacoemulsified (Figure 2). Multiple passes of the phaco tip can be made in order to adequately debulk and depressurize the intumescent cataract.

Once this is completed, the phaco handpiece is removed from the eye. OVD is then injected to refill the anterior chamber (Figure 3). Depending on how much of the nucleus is removed, which is dependent on how soft the intumescent

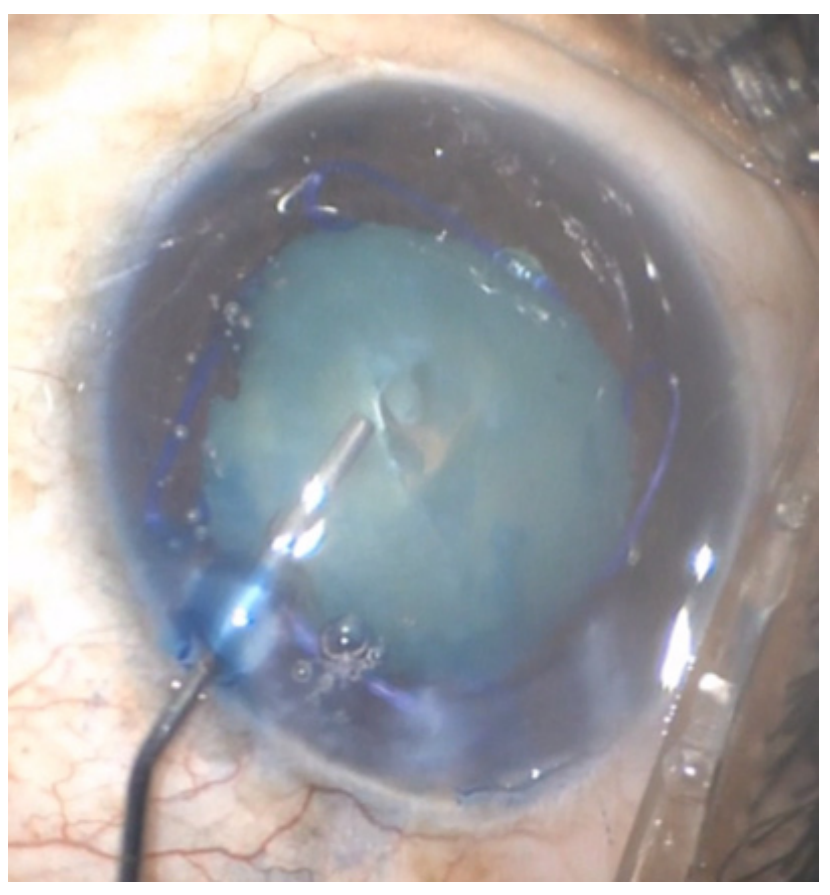

Figure 3 Ophthalmic viscosurgical device injected into the capsular bag and anterior chamber. 


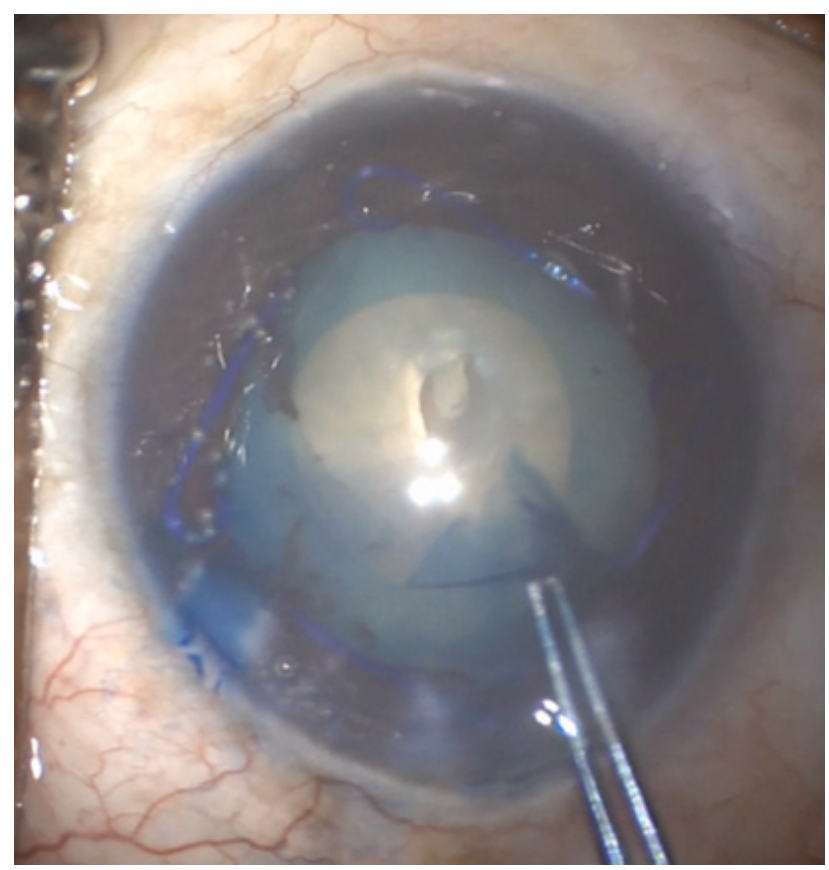

Figure 4 Completion of a continuous curvilinear capsulorhexis.

lens is, OVD may also need to be injected under the created anterior capsular opening to fill the capsular bag. Once the OVD is injected, completion of the CCC can be achieved by using capsulorhexis forceps (Figure 4). After the CCC, hydrodissection is performed and nucleus removal is completed with phacoemulsification, with the remainder of the procedure identical to routine cataract extraction.

The settings on the phacoemulsification machine should be set to the typical settings for sculpting the nucleus, which include low vacuum and moderate aspiration flow. While in position 3, either torsion or ultrasound may be used.

\section{Discussion}

With using a 27- or 30-gauge needle to remove liquefied cortex, it is possible that not enough liquefied cortex may be removed; thus, a spontaneous capsular tear may still occur. The advantage of using an automated phaco handpiece to aspirate the fluid instead of a needle is since the phaco handpiece is automated a large amount of fluid can be aspirated, in comparison with a needle. Additionally, an intumescent cataract may have different pockets of fluid, and an automated handpiece can reach and aspirate these various pockets more effectively than a needle. The 2-stage capsulorhexis can be time-consuming, is technically difficult to master, and also may not completely prevent the Argentinean Flag Sign. Femtosecond laser-assisted capsulotomy is effective in preventing capsulorhexis externalization, but cost and access to the laser may be a limiting factor.
Genç et al reported on 80 eyes that underwent phaco capsulotomy. ${ }^{8}$ They reported that phaco capsulotomy may reduce the risk for capsule tear, leading to safe cataract surgery in cases with intumescent cataracts. If not enough of the milky cortex is removed and depressurized, then the phaco capsulotomy may not be completely effective, and the Argentinean Flag Sign may still occur. Therefore, when performing the technique, adequate passes with the phaco handpiece must be performed to ensure that enough of the cataract is debulked.

An unpredictable feature of phaco capsulotomy is what type of opening is created in the anterior capsule when puncturing with the phaco handpiece tip. A capsulorhexis is typically initiated with a cystatome, and then a cystatome or capsulorhexis forceps is used to complete the capsulorhexis. After puncturing the anterior capsule with the phaco handpiece and aspirating the intumescent cataract, there is typically a jagged opening created in the capsulorhexis. After injecting OVD, the anterior capsule leaflets should be analyzed to see where best to grab and proceed with the capsulorhexis. There will always be an adequate leaflet to grab, and thereafter, a continuous capsulorhexis can be achieved.

Potential complications of phaco capsulotomy include directing too much force in a downward direction with the phaco probe and thus creating zonular dehiscence, or using too much vacuum power on the nucleus, thus pulling the lens toward the phaco tip and possibly creating zonular dehiscence. Additionally, wound burn can occur in the main wound. This occurs when the phaco tip embeds a dense nucleus, and there is rapid occlusion of the tip. To prevent this from occurring, the foot pedal can be released, and thereafter, the foot should step on and off the pedal, decreasing and increasing the vacuum in pulsed increments. Additionally, automated bursts of phaco energy may be programmed in the phacoemulsification machine, and a larger phaco handpiece tip may be used, to prevent occlusion.

Should the Argentinean Flag Sign still occur despite phaco capsulotomy, this means that there is still overbearing pressure in the intumescent cataract, and that not enough fluid was aspirated. An instrument can be used to push back the iris to try to visualize how far the extension propagated posteriorly. At this point, there are a few options. More OVD may be injected, and the capsulorhexis may be finished in the other direction. Another option is to aspirate more fluid from the cataract, either with the phaco handpiece or a syringe. Following this, more OVD can be injected and the capsulorhexis may be attempted to be completed in the opposite direction. The phacoemulsification should be commenced with caution. Since there is a capsulorhexis that has extended 
peripherally, it is optimal to first groove the nucleus and propagate a central crack, then proceed with gentle hydrodissection. During hydrodissection, the central crack allows the injected fluid to be released, so the fluid pressure does not further extend an impaired capsulorhexis. Finally, conversion to extracapsular cataract extraction is also an option.

Pearls for performing phaco capsulotomy include using a second instrument and using a $2.75-\mathrm{mm}$ wound or larger, and larger phaco tip. Eyes with mature intumescent lenses frequently have shallow anterior chambers, and when the phaco tip is introduced, the eye may be pushed to a distal position. A second instrument may be placed into the paracentesis to pull the eye back to an ortho position before the phaco capsulotomy is performed (Figure 1). A larger wound and phaco tip are also advantageous because there is less chance of the handpiece becoming occluded, thereby decreasing the risk of wound burn and facilitating removal of the dense lens fragments.

In conclusion, phaco capsulotomy is a safe and effective technique for preventing the Argentinean Flag Sign. By using the phaco tip to simultaneously create an anterior capsular puncture and remove the liquefied cortex and nucleus, the lens/capsule apparatus is decompressed and the impetus for the capsulorhexis to spontaneously tear outward is eliminated.

\section{Acknowledgment}

This work has been supported in part by an unrestricted/ challenge award to Yale Eye Center from the Research to Prevent Blindness (RPB), Inc.

\section{Disclosure}

The author reports no conflicts of interest in this work.

\section{References}

1. Perrone DM. Argentinean flag sign is the most common complication for intumescent cataracts. Ocul Surg News. US edition, December 15, 2000

2. Rao SK, Padmanabhan P. Capsulorhexis in eyes with phacomorphic glaucoma. J Cataract Refract Surg. 1998;24(7):882-884

3. Kara-Junior N, de Santhiago MR, Kawakami A, Carricondo P, Hida WT. Mini-rhexis for white intumescent cataracts. Clinics (Sao Paulo). 2009; 64(4):309-312.

4. Bissen-Miyajima H. Ophthalmic viscosurgical devices. Curr Opin Ophthalmol. 2008;19:50-54.

5. Conrad-Hengerer I, Hengerer FH, Joachim SC, Schultz T, Dick HB. Femtosecond laser-assisted cataract surgery in intumescent white cataracts. J Cataract Refract Surg. 2014;40(1):44-50.

6. Teng CC. Preventing the Argentinian flag sign: phaco capsulotomy. Eyeworld, February, 2013.

7. Mahalingam P, Sambhav K. Phaco capsulotomy in intumescent cataract. Nepal J Ophthalmol. 2014;6(2):242-243.

8. Genç S, Güler E, Çakır H, Özertürk Y. Intraoperative complications in intumescent cataract surgery using a phaco capsulotomy technique. $J$ Cataract Refract Surg. 2016;42(8):1141-1145.
Clinical Ophthalmology

\section{Publish your work in this journal}

Clinical Ophthalmology is an international, peer-reviewed journal covering all subspecialties within ophthalmology. Key topics include: Optometry; Visual science; Pharmacology and drug therapy in eye diseases; Basic Sciences; Primary and Secondary eye care; Patient Safety and Quality of Care Improvements. This journal is indexed on

\section{Dovepress}

PubMed Central and CAS, and is the official journal of The Society of Clinical Ophthalmology (SCO). The manuscript management system is completely online and includes a very quick and fair peer-review system, which is all easy to use. Visit http://www.dovepress.com testimonials.php to read real quotes from published authors. 